\title{
Getting nervous about IBD
}

\section{By Tim Fulmer, Senior Writer}

A paper published in The Journal of Clinical Investigation suggests that antidepressants used to treat psychological disorders often associated with gastrointestinal diseases may have the added therapeutic effect of reducing bowel inflammation. Harnessing this double effect to treat conditions such as ulcerative colitis and Crohn's disease will require determining which drugs are most efficient at achieving both therapeutic endpoints and whether such candidates would be used in place of, or on top of, existing treatments.

Common strategies for treating inflammatory bowel disease (IBD) involve suppressing the immune response with small molecules or antibodies that target proinflammatory cytokines. ${ }^{1}$ The major forms of IBD-ulcerative colitis (UC) and Crohn's disease-are characterized by inflammation of the large and small intestine. UC is usually restricted to the colon and rectum, and Crohn's disease can affect any part of the GI tract. ${ }^{2}$

IBD patients often suffer from psychological disorders such as stress and depression that may have a negative impact on their response to IBD therapy. ${ }^{3}$ However, it isn't known whether IBD patients suffer from depression prior to getting IBD or as a result of having IBD. Some researchers contacted by SciBX believe there is probably a bidirectional relationship between psychiatric disorders and inflammation.

Stephen Collins, a professor of medicine at McMaster University, and his coauthors on the JCI study believe their data suggest strategies for treating inflammation by modulation of the nervous system. This includes antidepressants that act on the brain or nicotinic receptor agonists that act on the vagus nerve. Both types of compounds, according to the team's data, could potentially alleviate psychiatric symptoms while also reducing inflammation in regions of the gut.

The researchers found that treating chemically induced colitis in mouse models of depression with a tricyclic antidepressant not only alleviated depressed behavior but also significantly reduced inflammation compared with what was seen in control mice $(p<0.05){ }^{4}$

Previous mouse data from Collins' lab had linked antidepressant therapy to reduced susceptibility to colitis ${ }^{5}$ and implicated the vagus nerve in protecting the colon from the disease. ${ }^{6}$ In the current paper, the researchers tested the hypothesis that antidepressants reduce colitis in depressed mice by restoring proper function of the vagus nerve in the gut. The vagus is a cranial nerve that innervates the pharynx, larynx, esophagus, heart and most GI organs.
The researchers found that the generic tricyclic antidepressant desmethylimipramine (also known as desipramine) reduced multiple parameters of colon inflammation in depressed mice without directly acting on the colon. Furthermore, vagotomy-severing the vagus nerve-abolished the anti-inflammatory activity of the compound in the same mice. Thus, the antidepressant effect of desipramine "is due to restoration of vagal parasympathetic function," the authors concluded.

\section{Targeting IBD in the brain}

Researchers contacted by SciBX agreed that antidepressants have therapeutic potential in the IBD space. However, they also said it will be important to determine the most efficacious types of antidepressants for achieving this combined psychological and inflammatory therapeutic effect and to determine how those molecules could be used with current treatment regimes.

"Although the data in our paper are not sufficient to advocate indiscriminate use of antidepressants to treat IBD, they do suggest that in cases where an IBD patient is depressed, attention should be paid to treating the depression in combination with treating the ulcerative colitis," said Collins.

Moreover, he thinks other types of antidepressants could have utility in IBD. "In the near future we want to investigate the antiinflammatory efficacy of selective serotonin reuptake inhibitors such as fluoxetine in our mouse models," Collins said. Prozac fluoxetine is a selective serotonin reuptake inhibitor (SSRI) marketed by Eli Lilly and Co. to treat depression.

Indeed, work by Antonina Mikocka-Walus and colleagues at Monash University has already shown that both desipramine and fluoxetine reduce circulating levels of proinflammatory cytokines and increase survival in rodent models of septic shock. ${ }^{7}$ The question, she said, is "whether some kinds of antidepressants are better than others" for treating inflammation that accompanies depression.

Mikocka-Walus also told SciBX a key issue that needs to be addressed is "whether we can replace standard anti-inflammatory therapies such as immunomodulators, 5-ASA drugs or prednisolone with antidepressants or whether they should be given on top of the standard treatment."

Next year, Mikocka-Walus hopes to begin a trial in Australia to evaluate the anti-inflammatory effects of an undisclosed antidepressant in patients with Crohn's or UC.

Satish Keshav, consultant gastroenterologist at John Radcliffe Hospital and a senior lecturer at Oxford University, said there is precedent for off-label use of antidepressants to treat GI disorders. "Intestinal inflammation associated with some forms of irritable bowel syndrome can be improved by treatment with the tricyclic antidepressant amitriptyline, though at a much lower dose than what treats depression," he said.

Irritable bowel syndrome (IBS) is a functional bowel disorder that presents without the tissue inflammation characteristic of IBD.

This month in The Journal of Pediatrics, researchers at the David Geffen School of Medicine at University of California, Los Angeles, 
published data from a double-blind trial showing that low doses of the generic depression drug amitriptyline significantly improved the quality of life of adolescent IBS patients compared with placebo. ${ }^{8}$

\section{Targeting the vagus}

In a separate set of experiments not involving antidepressants, the JCI paper showed that vagotomy was associated with decreased colonic tissue levels of acetylcholine, the primary neurotransmitter of the efferent vagus nerve. Thus, activation of acetylcholine receptors in the gut might help restore vagal function and also reduce colitis. Indeed, treatment of vagotomized mice with the acetylcholine receptor agonist nicotine significantly lowered inflammatory tissue damage compared with that in untreated controls $(p<0.05)$.

"Our paper supports a second therapeutic strategy for treating IBD-highly selective nicotinic receptor agonists," Collins told SciBX. "Previous work by Kevin Tracey and others has shown that stimulation of the vagus nerve releases the neurotransmitter
"Our paper supports a second therapeutic strategy for treating IBD-highly selective nicotinic receptor agonists."

-Stephen Collins, McMaster University macrophages or intestinal epithelium to help confirm the hypothesis that antidepressants act through the vagus to reduce intestinal inflammation.

Earlier this year, Stappenbeck and colleagues described a transforming growth factor- $\beta$ receptor and IL-10 receptor double-knockout mouse model that rapidly developed acute UC. ${ }^{15}$ Although treating the mice with antibodies against proinflammatory cytokines reduced disease severity, "it might certainly also be the case that giving the same models antidepressants could reduce inflammation," he said.

Stappenbeck added that with the recent publication of several UC and Crohn's disease susceptibility loci, ${ }^{16,17}$ "the opportunity arises to create a series of single- and multiple-gene knockout models that could help identify the key intestinal flora that triggers IBD and also characterize the efficacy of potential therapeutic strategies, including antidepressants and selective vagal nerve stimulation."

Collins plans to develop preclinical models of IBD that focus on intestinal flora.

"Key longer-term next steps include studying the bidirectional relationship acetylcholine, which subsequently binds to nicotinic receptors on macrophages and causes deactivation of those immune cells. Given that macrophages are a significant component of the IBD inflammatory response, it stands to reason that blocking their function in the gut could have considerable therapeutic value."

Tracey and colleagues have shown that exposing human tissue macrophages to nicotine or acetylcholine inhibits production of proinflammatory cytokines such as tumor necrosis factor (TNF), IL-1 and IL-18 while having little effect on anti-inflammatory cytokines such as IL-10.9,10 Tracey is CEO of the Feinstein Institute for Medical Research.

According to Ken Kengatharan, VP of translational research and scientific affairs at CoMentis Inc., one caveat is that nicotinic receptor agonists "may only be useful in ulcerative colitis and not Crohn's disease. There's a wealth of evidence that smokers have a lower incidence of ulcerative colitis but higher incidence of Crohn's."

For example, in two separately run double-blind trials, nicotine patches have been shown to be better than placebo at inducing remission in active UC. ${ }^{11,12}$ Furthermore, smoking cessation has been shown to improve the course of Crohn's. ${ }^{13,14}$

CoMentis has an oral agonist of the nicotinic acetylcholine receptor $\alpha 7$ subunit, GTS-21, in Phase IIa testing to treat attention deficit hyperactivity disorder (ADHD). Kengatharan told SciBX that "although the main focus of our GTS-21 program is in CNS disorders, we would like to consider the compound for IBD in the future."

\section{Model behavior}

Thaddeus Stappenbeck, assistant professor of pathology and immunology at Washington University School of Medicine, said, "The JCI paper represents an emerging field that needs to be recognized in the study of inflammatory disorders-neuroimmunology."

According to Stappenbeck, "key next steps would involve designing preclinical models to specifically characterize the molecular mechanism whereby antidepressants restore vagal function.” In particular, he suggested knocking out the nicotinic acetylcholine receptor on between depression and intestinal flora," he said. "On the one hand, there is evidence that psychological stress can affect the composition of intestinal flora. On the other hand, separate research has suggested that altering intestinal flora, as might occur with disease or change in diet, can not only result in gut inflammation but also act back on the CNS via neuroendocrine pathways to induce depression, stress and other psychiatric disorders.”

Lisa Goehler, professor of psychology and neuroscience at the University of Virginia, told SciBX the JCI paper shows that "at least one way antidepressants may inhibit inflammation is through the vagus nerve."

Using a Citrobacter rodentium-induced IBD mouse model, Goehler and colleagues have found that during the initial stages of infection, mice displayed considerably increased anxiety-like behavior even though plasma levels of inflammatory cytokines were unchanged and the colon showed no signs of inflammation. However, over the same period of time, vagal sensory neurons were increasingly active.

The authors concluded that the vagus nerve was "highly sensitive to the presence of potentially dangerous bacteria in the GI tract" and thus provided a link between intestinal flora and psychiatric states. $^{18}$

\section{REFERENCES}

1. Atreya, R. \& Neurath, M. Mucosal Immunol. 1, 175-182 (2008)

2. Xavier, R. \& Podolsky, D. Nature 448, 427-434 (2007)

3. Mikocka-Walus, A. BMC Gastroenterol. 7; published online Sept. 24, 2007; doi:10.1186/1471-230X-7-38

4. Ghia, J.-E. et al. J. Clin. Invest.; published online May 1, 2008; doi:10.1172/JCI32849

Contact: Stephen M. Collins, McMaster University, Hamilton, Ontario, Canada

e-mail: scollins@mcmaster.ca

5. Varghese, A. et al. Gastroenterology 130, 1743-1753 (2006)

6. Ghia, J.-E. et al. Gastroenterology 131, 1122-1130 (2006)

7. Roumestan, C. et al. Respir. Res. 8; published online May 3, 2007; doi:10.1186/1465-9921-8-35

8. Bahar, R. et al. J. Pediatr. 152, 685-689 (2008)

9. Tracey, K. Nature 420, 853-859 (2002) 
10. Oke, S. \& Tracey, K. J. Leukoc. Biol. 83, 512-517 (2008)

11. Pullan, R. et al. N. Engl. J. Med. 330, 811-815 (1994)

12. Sandborn, W. et al. Ann. Intern. Med. 126, 364-371 (1997)

13. Johnson, G. et al. Aliment. Pharmacol. Ther. 21, 921-931 (2005)

14. Thomas, G. et al. Nat. Clin. Pract. Gastroenterol. Hepatol. 2, 536-544 (2005)

15. Kang, S. et al. PLoS Med. 5, e41; published online March 4, 2008; doi:10.1371/journal.pmed.0050041

16. Fisher, S. et al. Nat. Genet.; published online April 27, 2008; doi:10.1038/ng.145

17. Franke, A. et al. Nat. Genet.; published online April 27, 2008; doi:10.1038/ng.148

18. Goehler, L. et al. Brain Behav. Immun. 21, 721-726 (2007)

\section{COMPANIES AND INSTITUTIONS MENTIONED}

CoMentis Inc., South San Francisco, Calif.

David Geffen School of Medicine, University of California, Los Angeles, Calif.

Eli Lilly and Co. (NYSE:LLY), Indianapolis, Ind.

Feinstein Institute for Medical Research, Manhasset, N.Y. John Radcliffe Hospital, Oxford, U.K.

McMaster University, Hamilton, Ontario, Canada

Monash University, Victoria, Australia

Oxford University, Oxford, U.K.

University of California, Los Angeles, Calif.

University of Virginia, Charlottesville, Va.

Washington University School of Medicine, St. Louis, Mo. 